\title{
Safenectomía corta versus larga en el tratamiento de las várices primarias de los miembros inferiores
}

\author{
Short versus long saphenectomy in the treatment primary inferior limb varices \\ Jesús Sánchez-Beorlegui ${ }^{1}$, Álvaro Arribas ${ }^{1}$, Antonio Aspíroz² ${ }^{2}$ Laura Lamata ${ }^{3}$, Félix Lamata ${ }^{3}$, \\ Alfredo Jiménez ${ }^{3}$
}

Servicio de Cirugía General y Flebología, Hospital Viamed Montecanal, Zaragoza, España
Servicio de Anestesiología, Hospital Viamed Los Manzanos, Zaragoza, España
Servicio de Cirugía General y Digestiva, Hospital Clínico Universitario Lozano Blesa, Zaragoza, España

\section{Resumen}

Introducción. El limitar la longitud de la extirpación (stripping) de la vena safena mayor al segmento insuficiente con base en los hallazgos de la ecografía Doppler a color, permite disminuir la morbilidad.

Materiales y métodos. Se llevó a cabo un estudio prospectivo y aleatorio de 155 pacientes intervenidos durante 24 meses, de los cuales 74 fueron sometidos a una safenectomía corta y 8I a una safenectomía larga. Las variables evaluadas durante el seguimiento fueron: tipo de anestesia, porcentaje de atención ambulatoria, morbilidad quirúrgica, días de incapacidad temporal y recidivas varicosas. Los síntomas de afectación neurológica ligados a la safenectomía se evaluaron de forma temprana y a largo plazo.

Resultados. El 91,6\% de los enfermos fueron intervenidos de forma ambulatoria, con un período de incapacidad temporal menor de tres semanas y $18 \%$ de recidivas. El costo unitario de las intervenciones concertadas con el Sistema Público de Salud fue inferior a USD\$950. La safenectomía corta tuvo mejores resultados que la larga en casi todos los parámetros estudiados, con menos días de ausencia laboral (I8,2 Vs. 22,6; p=0,ol3), morbilidad (I4,9 $\%$ Vs. 33,3\%; $\mathrm{p}=0,036)$ y secuelas neurológicas a la finalización del estudio (2 \% Vs. II,9 \%; $\mathrm{p}=0,043)$.

Discusión. La safenectomía clásica es una técnica útil, con excelentes resultados clínicos y estéticos, escasas complicaciones y bajo costo. En ausencia de insuficiencia distal del eje safeno, la safenectomía corta es de elección por su menor morbilidad.

Palabras clave: várices; vena safena; insuficiencia venosa; procedimientos quirúrgicos vasculares; complicaciones posoperatorias; recurrencia.

\footnotetext{
Abstract

Background: Limited stripping from the great saphenous vein to the insufficient segment based on the Doppler color echography findings reduces morbidity.

Material and methods: A prospective randomized study was conducted on 155 patients who underwent surgery over a 24 months period, of which 74 were subjected to limited saphenectomy and 8 I to total saphenectomy. The

Fecha de recibido: 16/01/2018 - Fecha aceptación: 1/02/2018

Correspondencia: Jesús Sánchez-Beorlegui, MD, Calle Ortega y Gasset No 6, $4^{\circ}$ C. 26007, Logroño (La Rioja), España / Teléfonos: (0034941) 500-587 y (608) 160-584

Correo electrónico: jsbeorlegui@gmail.com

Citar como: Sánchez-Beorlegui J, Arribas A, Aspíroz A, Lamata L, Lamata F, Jiménez A. Safenectomía corta versus larga en el tratamiento de las várices primarias de los miembros inferiores. Rev Colomb Cir. 2018;33:181-8. https://doi.org/10.30944/20117582.60
} 
monitored variables during the followed up were: type of anesthesia, percentage as ambulatory surgery, surgical morbidity, temporary disability days, and recurrent varicose veins. Symptoms of neurological disturbances linked to saphenectomy were evaluated early and also at long term followup.

Results: 91.6\% were operated on as outpatients, exhibiting shorter period of temporary disability (3 weeks) and I8\% recurrence rate. The cost of the actions agreed with the Public Health System interventions tariff, less than \$950 USD. The limited saphenectomy procedure appeared better than the total saphenectomy in almost all parameters studied, with fewer days of sick leave (I8,2 vs $22,6 \mathrm{p}=0.013)$, morbidity rate ( $14.9 \% \mathrm{vs} 33.3 \% \mathrm{p}=0.036$ ), and neurological sequelae at completion of the study ( $2 \%$ vs II. $9 \% \mathrm{p}=0.043)$.

Discussion: The classical saphenectomy is a useful technique, with excellent clinical and cosmetic results, few complications and low cost. In the absence of distal saphenous insufficiency axis, the limited saphenectomy appears as the preferred procedure in view of its lower morbidity.

Key words: varicose veins; saphenous vein; venous insufficiency; vascular surgical procedures; postoperative complications; recurrence.

\section{Introducción}

Pese a la progresiva implantación de las técnicas de ablación endovascular, la safenectomía quirúrgica no ha perdido su utilidad ni vigencia en el tratamiento de las várices de las extremidades inferiores ${ }^{1,2}$. La actualización que los cirujanos hemos llevado a cabo de la safenectomía clásica, ha permitido minimizar sus desventajas teóricas (mayor morbilidad, incisión inguinal, incapacidad laboral prolongada, estancia hospitalaria), potenciando sus virtudes, demostradas a lo largo de decenios de aplicación: sencillez, reproducibilidad, mínima inversión en aparataje y bajo costo, además de unos resultados clínicos homologables sustentados en estudios a largo plazo ${ }^{3}$. Por todo ello, la safenectomía quirúrgica continúa siendo hoy en día el método de referencia para comparar cualquier nueva técnica, y una opción válida para tratar satisfactoriamente al paciente con várices ${ }^{\mathrm{I}, 4}$.

Una de las aportaciones más interesantes en estos últimos años ha sido limitar la longitud de la extirpación de la vena safena mayor al segmento insuficiente, con base en la ecografía Doppler a color ${ }^{5,6}$. Las principales ventajas de esta safenectomía parcial o corta frente a la completa, larga o inguino-maleolar, serían la disminución de las secuelas neurológicas, la preservación de un segmento safeno en previsión de futuras cirugías cardiacas, mejor recuperación y reincorporación precoz al trabajo ${ }^{6-8}$.
En el presente estudio, se evaluaron los resultados de la safenectomía corta frente a la larga en un grupo homogéneo de pacientes intervenidos por várices, junto con los beneficios que las modificaciones diagnósticas, técnicas y anestésicas implementadas han aportado a la safenectomía clásica.

\section{Materiales y métodos}

En el estudio se incluyeron pacientes afectos de várices, derivados desde el Sistema Público de Salud al Servicio de Cirugía General y Flebología del Hospital Viamed Montecanal y tratados mediante safenectomía. Su duración fue de 24 meses, de septiembre de 2012 a octubre de 2014 . Los controles para la evaluación de los resultados y la morbilidad a largo plazo, se programaron, como mínimo, un año después del acto operatorio y finalizaron en junio de 2016.

\section{Diseño}

Se trató de un estudio prospectivo aleatorizado de forma equilibrada, asignando un número similar de sujetos a cada grupo del ensayo mediante la aplicación alternativa de cada una de las técnicas estudiadas, o bien, la utilización de una misma técnica a lo largo de una sesión quirúrgica completa. Los procedimientos diagnósticos, quirúrgicos y el seguimiento fueron llevados a cabo por el mismo facultativo, minimizando el factor de dependencia del cirujano. 
Las variables registradas fueron las siguientes:

- Primera cita en consulta y en las revisiones durante los dos primeros meses: epidemiología, tipo de anestesia, tratamiento ambulatorio, morbilidad quirúrgica (alteraciones cutáneas, infección, hematoma, trombosis superficial), síntomas tempranos de afectación neurológica y duración de la incapacidad laboral.

- Revisión al año: presencia de recidiva varicosa (incluyendo clasificación y tratamiento propuesto) y secuelas neurológicas.

\section{Selección de casos}

Los criterios de inclusión fueron: várices sintomáticas con insuficiencia ostial del cayado de la vena safena mayor y dilatación en el muslo, establecidas mediante la evidencia clínica y ecografía Doppler a color; clasificación clínica, etiológica, anatómica y fisiopatológica (CEAP) (International Consensus Committee on Venous Disease várices tronculares (C2), con o sin edema de miembros inferiores $\left(\mathrm{C}_{3}\right)$ o signos de pigmentación o eccema (C4a), de etiología primaria (Ep), que afectaran en exclusiva al sistema venoso superficial (As) y cuya fisiopatología se debiera a un reflujo ocasionado en las válvulas venosas insuficientes (Pr); competencia distal de la safena mayor con ausencia de dilatación o reflujo en los tercios medial y distal de la pierna durante el estudio Doppler; y pacientes laboralmente activos.

Los criterios de exclusión fueron: cirugía venosa previa en la extremidad o cirugía bilateral; estado de anticoagulación o antiagregación, y antecedente de trombosis venosa profunda.

\section{Protocolo diagnóstico}

En el transcurso de la primera consulta, se elaboró la historia clínica, se hizo la exploración visual y con ecografía Doppler a color de las extremidades, se firmó el consentimiento informado y se solicitó el estudio preoperatorio.

\section{Técnica anestésica}

Las técnicas empleadas fueron la anestesia espinal unilateral con bupivacaína hiperbárica ${ }^{9}$, la anestesia general intravenosa con propofol y re- mifentanilo asociada a la inserción de una mascarilla laríngea ${ }^{\mathrm{IO}} \mathrm{y}$, entre las técnicas combinadas, la sedoanalgesia complementada con una anestesia local tumescente del trayecto de la vena safena mayor utilizando una dilución de bupivacaína, bicarbonato y epinefrina en suero salino ${ }^{\mathrm{II}, 12}$.

\section{Técnica quirúrgica}

Se practicó crosectomía reglada a través de una incisión de dos $\mathrm{cm}$ por debajo del pliegue inguinal y, además, ligadura de las colaterales, sutura por transfixión del muñón safeno con material no absorbible y disección del tronco de la safena mayor en el tercio superior del muslo para desconectar las safenas accesorias y la comunicante de Giacomini.

A continuación, se practicó una safenectomía larga hasta el maléolo interno o una safenectomía corta hasta el tercio superior o medio de la pierna. La fleboextracción se practicó según la técnica descrita por van der Stricht o mediante extracción con un PIN-Stripper ${ }^{13,14}$, llevando a cabo la invaginación sobre un hilo largo de seda gruesa o la guía plástica del kit de safenectomía ${ }^{13,15,16}$.

Tras extirpar la vena safena mayor, se procedió a tratar las perforantes insuficientes y colaterales dilatadas mediante flebectomía segmentaria ${ }^{17}$, a la vez que se colocó el miembro inferior con una elevación de $30^{\circ}$ para favorecer el drenaje venoso.

Una vez suturadas las incisiones con hilo monofilamento de 4 ceros, se colocó un vendaje tricapa (compresas empapadas en suero, Velband ${ }^{\circ}$ y venda autoadhesiva) confeccionado, en sentido de distal a proximal, para evacuar los restos hemáticos. Tras el vendaje, se procedió al cierre por planos de la incisión inguinal, con especial atención a la oclusión del canal safeno con sutura reabsorbible. El vendaje fue sustituido en la consulta de enfermería entre las 48 y 72 horas por una media elástica de compresión tipo Farmalastic Novum Intelligent ${ }^{\circ}$ o Mediven Struva ${ }^{\circ}$ 23-35.

\section{Descripción de las complicaciones}

Las anomalías neurológicas se agruparon según la clasificación de Morrison y Dalsing ${ }^{18}$ en: estadio I, entumecimiento o sensibilidad reducida 
en la pierna, tobillos o región gemelar; estadio 2, parestesias, disestesias o ambas; estadio 3, dolor neurógeno intenso, brusco o lacerante en la pierna, en el dorso del pie o en ambos; estadio 4, otros síntomas; y estadio 5, ninguno de los anteriores.

Para describir las recidivas, se empleó la clasificación propuesta por Perrin, et al., en su estudio REVAS ${ }^{19} \mathrm{y}$, para la nomenclatura flebológica, se adoptaron los consensos internacionales ${ }^{20}$.

\section{Estadística}

En la comparación de las variables clínicas y los resultados obtenidos, para las variables cualitativas se utilizó la prueba de ji al cuadrado (test exacto de Fisher) con la corrección de Yates para muestras pequeñas $(<200) \mathrm{y}$, para las cuantitativas, la prueba t de Student de comparación de medias. En ambas pruebas, el nivel de significación para $\mathrm{p}$ fue menos de 0,05; se consideró muy significativo un valor de p menor de o,oI. El tratamiento estadístico de los datos se hizo con el programa informático G-Stat 2.0 ${ }^{\mathrm{TM}}$.

\section{Resultados}

Durante los 24 meses del estudio, se incluyeron I55 pacientes que cumplieron con los criterios de selección, 74 de los cuales se sometieron a una safenectomía corta y, 8I, a una safenectomía larga. La muestra se obtuvo de un total de 438 pacientes intervenidos por várices durante el periodo de estudio.
La edad media fue de 40,6 años (rango de I9 a 68) y la distribución por sexo fue de $58 \%$ hombres y $42 \%$ mujeres.

En cuanto a la ocupación, en esta serie hubo $46,5 \%$ de funcionarios y asalariados por cuenta ajena (72 casos) frente a 53,5\% de trabajadores autónomos y por cuenta propia (83 casos). En este segundo grupo, se incluyeron 17 jubilados que mantenían una actividad profesional de tipo agrario o vinculada a negocios familiares.

La clasificación CEAP del tipo de várices en los $\mathrm{I}_{5} 5$ casos operados fue: $\mathrm{C}_{2}$ en 57 (36,8 \%), C3 en $85(54,8 \%)$ y C4a en I3 $(8,4 \%)$. En total, se practicaron 86 safenectomías en la extremidad inferior izquierda y 69 en la derecha.

La intervención se llevó a cabo mediante anestesia intradural (local y regional) en 134 pacientes $(86,4 \%)$, general (mascarilla laríngea) en $8(5,2 \%)$, y local tumescente más sedación en i3 $(8,4 \%)$.

Un total de 142 (9I,6 \%) pacientes fueron intervenidos en el régimen de cirugía mayor ambulatoria. En I3 casos se requirió hospitalización durante una noche: cuatro, por no cumplir los criterios para cirugía ambulatoria (distancia al hospital); siete, por no reunir los criterios de egreso (retención urinaria en 3 , ventana de recuperación corta en 2, dolor no controlado en I, e intolerancia alimentaria en I), y dos, por negativa a la cirugía ambulatoria. No se produjo ningún reingreso.

En la tabla I, se muestra la morbilidad temprana, durante los dos primeros meses, y en la

Tabla 1. Duración de la incapacidad temporal y morbilidad a corto plazo de la safenectomía corta versus larga

\begin{tabular}{lccc}
\hline & SC $(\mathbf{n}=74)$ & SL $(\mathbf{n}=81)$ & p \\
\hline Días de incapacidad temporal & $18,2(10-21)$ & $22,6(18-50)$ & $\mathrm{t}, \mathrm{p}=0,013$ \\
(media, mínimo y máximo), $\mathrm{n}(\%)$ & $10 / 74(14,9)$ & $22 / 81(33,3)$ & $\chi^{2}, \mathrm{p}=0,036$ \\
Morbilidad general, $\mathrm{n}(\%)$ & 3 & 4 & \\
Alteraciones cutáneas y edema & 1 & 2 & \\
Infección & 7 & 9 & \\
Hematoma inguinal, en muslo o ambos & 1 & 1 & \\
Trombosis superficial & $2(2,7)$ & $12(14,8)$ & $\chi^{2}, \mathrm{p}=0,008$ \\
\hline
\end{tabular}

SC: safenectomía corta; SL: safenectomía larga 
tabla 2, la morbilidad definitiva registrada al finalizar el seguimiento.

Se hizo un estudio específico de la duración de la incapacidad temporal, relacionándola con la modalidad de contratación y el tipo de safenectomía.

La comparación entre funcionarios y trabajadores por cuenta ajena versus autónomos y trabajadores por cuenta propia, mediante la prueba t de Student, mostró una diferencia muy significativa de 27,058 días frente a I5,23 (p=0,000I) (figura I).

Sin embargo, al analizar las dos modalidades de safenectomía en los trabajadores autónomos y por cuenta propia ( 83 casos: 39 con safenectomía corta y 44 con safenectomía larga), mediante la prueba t de Student, no se demostraron diferencias estadísticas: $\mathrm{I} 4,25$ días la corta frente a I6,I4 días la larga ( $\mathrm{p}=0,067)$.

Estas diferencias sí fueron significativas al comparar ambas técnicas, mediante la prueba $t$ de Student, en el grupo de los funcionarios y trabajadores por cuenta ajena ( 72 casos, 35 safenectomía corta más 37 safenectomía larga): 23,19 días la safenectomía corta frente a 30,50 días la larga ( $\mathrm{p}=\mathrm{0}, \mathrm{ooo} 6)$.

Un total de II8 pacientes acudieron a la revisión anual, por lo que se perdió el $24 \%$ de los casos para el estudio.

De las 2I recidivas registradas, la mayoría fueron microvárices (telangiectasias y várices reticulares) localizadas en la zona del complejo de Boyd, la cara interna del muslo o la región pretibial. En I9 pacientes se usó exclusivamente la esclerosis con espuma ${ }^{17,21} \mathrm{y}$ tan sólo dos tuvieron que ser reintervenidos: uno por un remanente largo del cayado que originaba una 'neosafena' en el muslo y otro por una recidiva serpiginosa en la cara interna de la pierna sobre una vena de Leonardo.
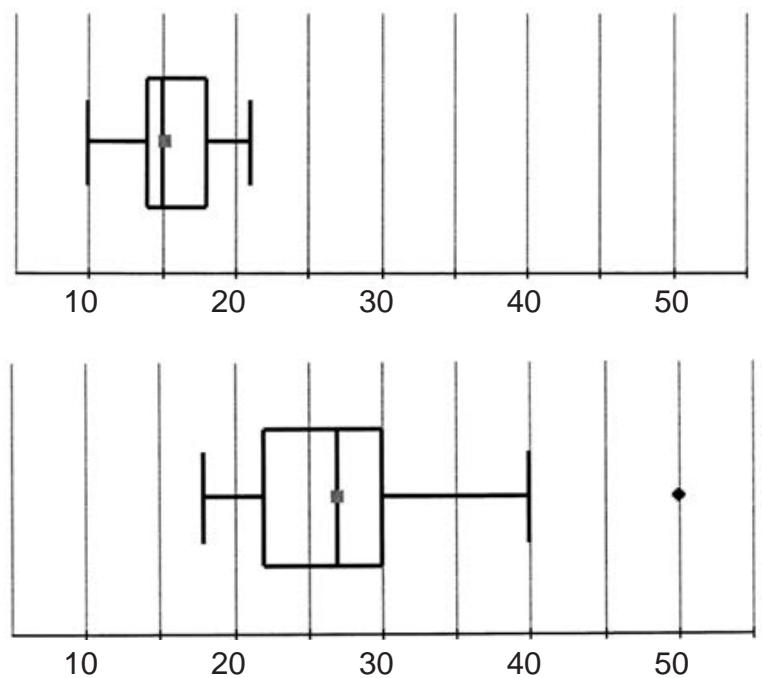

Figura 1. Comparación de los gráficos de caja con la distribución de los días de incapacidad temporal tras la safenectomía: en la parte superior, el box-plot de los autónomos y trabajadores por cuenta propia, en la inferior, funcionarios y trabajadores por cuenta ajena

Tabla 2. Resultados y morbilidad a largo plazo de la safenectomía corta versus larga

\begin{tabular}{llll}
\hline & \multicolumn{1}{c}{ SC (n=51) } \\
$\mathbf{n}(\%)$ & $\begin{array}{c}\text { SL }(\mathbf{n}=67) \\
\mathbf{n}(\%)\end{array}$ & $\mathbf{p ~}$ \\
\hline Recidivas varicosas & $8(15,7)$ & $13(19,4)$ & $\chi^{2}, p=0,601$ \\
Muslo & 2 & 2 & \\
Pierna & 5 & 7 & \\
Muslo y pierna & 1 & 4 & \\
Secuelas estéticas & $3(5,8)$ & $2(3)$ & $\chi^{2}, p=0,438$ \\
Hiperpigmentaciones en el muslo & 1 & 1 & \\
Cicatrices hipertróficas o antiestéticas & 2 & - & \\
Desarrollo de microvárices y telangiectasias (matting) & - & 1 & \\
Secuelas neurológicas definitivas & $1(2)$ & $8(11)$ & $\chi^{2}, p=0,043$ \\
\hline
\end{tabular}

SC: safenectomía corta; SL: safenectomía larga 


\section{Discusión}

La cirugía de las várices es la segunda intervención más frecuente en cirugía general y con una demanda creciente ${ }^{\mathrm{I}}$. El afrontar esta realidad supone un reto para la sanidad pública debido a la necesidad de contener el gasto que suponen la inversión en equipos de última generación y las ausencias laborales prolongadas. Nuestra casuística demuestra que la cirugía clásica puede hacer frente a estos objetivos de una manera satisfactoria con una tasa de cirugía ambulatoria del $90 \%$, incapacidad temporal inferior a las tres semanas, morbilidad neurológica definitiva del $7 \%$ y, a largo plazo, un porcentaje de recidivas del I $8 \%$, inferior a lo publicado en la literatura (20-28\%) ${ }^{19}$, además de óptimos resultados estéticos (tablas I y 2). Estas prestaciones son equiparables a las de las técnicas de ablación endovascular ${ }^{3,22}$.

En cuanto a la técnica de la safenectomía, nuestra experiencia demuestra las bondades de determinados actos:

- Se hace una meticulosa marcación con un dermorrotulador en el periodo prequirúrgico, con asistencia de un ecógrafo Doppler portátil.

- Mínima incisión por debajo del pliegue inguinal; ligadura de las colaterales visibles, pero sin disecciones amplias que favorezcan los seromas con gran riesgo de sobreinfección ${ }^{23}$; ligadura de la safena accesoria anterior y de la vena de Giacomini, más oclusión del canal safeno con un punto reabsorbible para prevenir la formación de hematomas inguinales.

- En la safenectomía corta, el fleboextractor se dirige de la ingle hacia el maléolo y, en la safenectomía larga ascendente, en sentido contrario. Cuando hay válvulas o tortuosidades que impidan la progresión de la guía, se diseca la vena safena mayor en la zona marcada con ecografía, para dirigirla o insertar otro stripper para una safenectomía en tramos ${ }^{8,16}$.

- Desconexión de tributarias y perforantes, tensionando el stripper en ambos extremos y haciendo maniobras de tracción o contratracción a lo largo del trayecto de la vena safena mayor ${ }^{\mathrm{II}, 13}$, lo que favorece la invaginación de la vena y reduce el riesgo de lesionar los nervios sensitivos ${ }^{16}$. Esta maniobra nos parece más importante que la dirección del stripping ${ }^{24}$.

- Los paquetes varicosos del muslo y en el complejo de Boyd, deben extirparse y no sólo desconectarse de la vena safena mayor. Sin embargo, desde que se implementó la ablación mecánico-química (MOCA) con el dispositivo Clarivein ${ }^{\circledast}$ en la Unidad, hemos dejado de practicar flebectomías complementarias durante el acto quirúrgico.

- Cuando el sangrado por el canal safeno no cede con la elevación de la extremidad, el vendaje compresivo tricapa "húmedo" nos ha ofrecido muy buenos resultados en la prevención de hematomas. Debe retirarse en 48 a 72 horas y sustituirse por una media elástica ${ }^{25,26}$. Sin embargo, en la mayoría de los casos, la media elástica se coloca en el quirófano ${ }^{26}$.

La comparación entre la safenectomía corta y la larga muestra que la primera presenta menor morbilidad general (tablas I y 2) y síntomas neurológicos netamente inferiores a corto y largo plazo ${ }^{7,8}$, aunque se debe reseñar que todos los pacientes manifestaron síntomas de tipo i o 2 de Morrison y Dalsing, sin restricciones importantes para sus actividades diarias.

En este estudio, se ha intentado evaluar la duración de la incapacidad temporal tras una safenectomía quirúrgica (tabla I, figura I) constatando las siguientes 'evidencias':

- La media del número de días que tardan nuestros pacientes para reincorporarse al puesto de trabajo, es menor que la prevista por las autoridades sanitarias que establecen un periodo de 20 a 30 jornadas para esta intervención ${ }^{27}$.

- Los pacientes sometidos a una safenectomía corta retornan antes a su actividad en todas las situaciones analizadas. Sin embargo, en el caso de los trabajadores autónomos y por cuenta propia, las diferencias no son significativas con respecto a los sometidos a una safenectomía larga: parece indudable que la necesidad de atender los negocios propios o 
generar recursos económicos, es un estímulo para acortar el período de ausencia laboral.

- La modalidad del desempeño laboral es un factor clave en la duración del periodo de incapacidad temporal. Comparando los box-plot en la figura I, resulta evidente que los funcionarios y trabajadores por cuenta ajena tienden a prolongar su recuperación, por lo que el diagrama muestra un rango intercuartílico mucho más amplio y una distribución asimétrica de los datos (desplazamiento de la mediana hacia la derecha de la caja y longitud del 'bigote' (whisker) del tercer cuartil). En este sentido, se detectaron incapacidades que se alargaron cuatro o cinco semanas en safenectomías no complicadas, pese al control por atención primaria y el régimen de prestaciones mutuas.

La cirugía de las várices ha experimentado una demanda creciente y un aumento de los costos derivado del uso de las técnicas endovasculares de oclusión venosa térmica (láser, radiofrecuencia o vapor de agua) o MOCA con Clarivein ${ }^{\circledast}$. Actualmente, la tarifa en la medicina privada de una ablación oscila entre los USD\$ 2.500 y los USD \$ 4.00o, que se reducen a USD\$ I.500-2.00o en el caso de una safenectomía. Como consecuencia, la mayoría de los servicios públicos de salud a nivel mundial excluyen la ablación de su lista de coberturas asistenciales gratuitas 1,28 .

En el caso de una safenectomía quirúrgica unilateral ambulatoria concertada con el Sistema Público Aragonés de Salud, nuestra Clínica factura USD\$950, incluidos los honorarios del cirujano y del anestesista, consulta, estudio preoperatorio, material fungible y gastos de hospitalización; esto, en vista de las buenas prestaciones que se han obtenido y su bajo costo, le permiten mantener su vigencia.

Como conclusión, la safenectomía clásica sigue siendo una técnica útil, barata y con excelentes resultados. En ausencia de insuficiencia distal del eje safeno interno, la safenectomía corta es electiva por su menor morbilidad. El seguimiento de la incapacidad temporal tras la cirugía es un aspecto que debería preocupar al Sistema Público de la Seguridad Social. La mejora de las medidas de control y establecer incentivos, permitirían reducir el ausentismo y ahorrar muchas jornadas laborales que se pierden en bajas no justificadas desde el punto de vista clínico.

\section{Referencias}

I. Morales-Cuenca G, Moreno-Egea A, Aguayo-Albasini JL. Los cirujanos generales frente a la cirugía de las várices. Cir Esp. 2009;85:205-13.

2. Gómez-Palacio VM, Águila-Márquez R. Cirugía venosa de mínima invasión. Cir Cir. 2010;78:497-504.

3. Rasmussen LH, Lawaetz M, Bjoern L, Blemings A, Eklof B. Randomized clinical trial comparing endovenous laser ablation and stripping of the great saphenous vein with clinical and duplex outcome after 5 years. J Vasc Surg. 20I3;58:42I-6.

4. Rasmussen LH, Bjoern L, Lawaetz M, Blemings A, Lawaetz B, Eklof B. Randomized trial comparing endovenous laser ablation of the great saphenous vein with high ligation and stripping in patients with varicose veins: Short-term results. J Vasc Surg. 2007;46:308-I5.

5. Kostas TT, Ioannou CV, Veligrantakis M, Pagonidis C, Katsamouris AN. The appropriate length of great saphenous vein stripping should be based on the extent of reflux and not on the intent to avoid saphenous nerve injury. J Vasc Surg. 2007;46:1234-4I.

6. Herman J, Lovecek M, Svach I, Duda M. Limited versus total stripping of vena saphena magna. Bratisl Lek Listy. 2002;103:434-6.

7. Flu HC, Breslau PJ, Hamming JF, Lardenoye JW. A prospective study of incidence of saphenous nerve injury after total great saphenous vein stripping. Dermatol Surg. 2008;34:1333-9.

8. Irace L, Siani A, Laurito A, Cappello F, Brunetti M, Tozzi M. Stripping corto della vena grande safena (Risultati ed indicazioni). Minerva Cardioangiol. 200I;49:383-8.

9. Del Río Fernández S. Anestesia espinal en cirugía mayor ambulatoria: ¿Estamos en el camino de la técnica anestésica ideal para procedimientos ambulatorios? Cir May Amb. 2013;18:182-6.

Io. Zaballos M, López S. Recomendaciones prácticas de uso de la mascarilla laríngea en cirugía ambulatoria. Cir May Amb. 2008;13:4-26.

II. Proebstle TM, Paepcke U, Weisel G, Gass S, Weber L. High ligation and stripping of the long saphenous vein using the tumescent technique for local anesthesia. Dermatol Surg. 1998;24:149-53.

I2. Bush RG, Hammond KA. Tumescent anaesthetic technique for long saphenous stripping. J Am Coll Surg. I999;189:626-8. 
13. Conrad P, Gassner P. Invagination stripping of the long and short saphenous vein using the PIN stripper. Aust N Z J Surg. 1996;66:394-6.

I4. Dieudonne G. Les saphénectomies par invagination sur fil sous anesthésie locale (technique de J. Van der Stricht modifiée). Phlébologie. 1988;41:309-20

I5. Goren G, Yellin AE. Invaginated axial saphenectomy by a semirigid stripper: Perforate-invaginate stripping. J Vasc Surg. 1994;20:970-7.

16. Scheltinga MR, Wijburg ER, Keulers BJ, de Kroon $\mathrm{KE}$. Conventional versus invaginated stripping of the great saphenous vein: A randomized, double-blind, controlled clinical trial. World J Surg. 2007;31:2236-42.

I7. Córdova P, Flores M, Zárraga JR. Eficacia del manejo en la enfermedad venosa crónica con escleroterapia guiada por USG y crosectomía comparadas con safenectomía convencional en el Servicio de Angiología y Cirugía Vascular, en el Hospital Regional Lic. Adolfo López Mateos. Rev Mex Angiol. 2013;41:25-9.

I8. Morrison C, Dalsing MC. Signs and symptoms of saphenous nerve injury after greater saphenous vein stripping: prevalence, severity, and relevance for modern practice. J Vasc Surg. 2003;38:886-90.

19. Perrin MR, Labropoulos N, Leon LR. Presentation of the patient with recurrent varices after surgery (REVAS). J Vasc Surg. 2006;43:327-34.

20. Gómez C, Jiménez H, Ulloa JH. Nomenclatura de las venas de los miembros inferiores y términos en flebología: los consensos internacionales. Rev Colom Cir. 2012;27:139-45.

2I. Tessari L, Cavezzi A, Frullini A. Preliminary experience with a new sclerosing foam in the treatment of varicose veins. Dermatol Surg. 200I;27:58-6o.
22. Rautio T, Ohinmaa A, Perala J, Ohtonen P, Heikkinen T, Wiik $\mathrm{H}$, et al. Endovenous obliteration versus conventional stripping operation in the treatment of primary varicose veins: A randomized controlled trial with comparison of the costs. J Vasc Surg. 2002;35:958-65.

23. Casoni P, Lefebvre-Vilardebo M, Villa F, Corona P. Great saphenous vein surgery without high ligation of the saphenofemoral junction. J Vasc Surg. 20I3;58:I73-8.

24. Milone M, Di Minno MN, Maietta P, Shatalova O, Musella M, Milone F. Great saphenous vein stripping and nerve injury: The role of stripping direction. Int Angiol. 2015;34:238-42.

25. Collazo E, Luque MA, González-Ripoll C. Terapia compresiva tras cirugía de várices. ¿Es mejorable el vendaje elástico clásico? Cir Esp. 2010;88:232-7.

26. Huang TW, Chen SL, Bai CH, Wu CH, Tam KW. The optimal duration of compression therapy following varicose vein surgery: A meta-analysis of randomized controlled trials. Eur J Vasc Endovasc Surg. 2013;45: 397-402.

27. Manual de tiempos óptimos de incapacidad temporal. Tercera edición. Instituto Nacional de la Seguridad Social 2013. Fecha de consulta: 20 de mayo de 2016. Disponible en: http://www.seg-social.es/prdioo/groups/ public/documents/binario/I78382.pdf.

28. Hong KP. Midterm clinical outcomes after modified high ligation and segmental stripping of incompetent small saphenous veins. Korean J Thorac Cardiovasc Surg. 2015;48:398-403. 